et al, 1987). It is also interesting to compare this result with Selwyn's finding of increased needle-sharing and reduced knowledge about HIV in intravenous drug users seen at a non-treatment site (Selwyn et al, 1987), since in the present study the differences are observed between clients attending two different types of treatment centre.

Longitudinal studies would be needed to determine whether the samples of drug users remain distinct or if the differences found represent points in a fluctuating career of drug dependence. With this caveat, the finding of more needle sharing behaviour within three months at the satellite clinic implies that this clinic is bringing intravenous drug users in greater need of health education and counselling into contact with health and treatment services. Probation services probably have a high level of contact with drug users (Tirrelli et al, 1986; Selwyn et al, 1987) yet treatment services have been slow to form therapeutic links. The clinic described here provides a low cost model of a service which is demonstrably effective in reaching a high risk group and so is ideally suited to a major role in countering the HIV epidemic.

\section{References}

Daviaud, E. (1987) Report on Rathbone Place Weekly Clinic. The 1986 Referrals. Drug Indicators Project,
Birkbeck College, University of London, 1987 [Unpublished report].

Dolans, M. P., Black, J. L., Deford, H. A., Skinner, J. R. \& RoBinowITZ, R. (1987) Characteristics of drug abusers that discriminate needle sharers. Public Health Reports, 395-398.

Harms, G., Laukamm-Josten, U., Bienlze, U. \& GUGGENMOOS-HolyMANN, I. (1987) HIV antibodies in German IV drug users. Kiln Wochenschur, 65, 367-379.

Hartnoll, R., Lewis, R., Mitcheson, M. \& BREWer, S. (1985) Estimating the prevalence of opioid dependence. The Lancet, i, 203-205.

MulleAdY, G. \& GREEN, J. (1985) Syringe sharing among London drug abusers. The Lancet, ii, 1425.

Robertson, J. R., Bucknall, A. B. V. \& WigGun, P. (1986) Regional variations in HIV antibody seropositivity in British intravenous drug users. The Lancet, ii, 1435-1436.

Selwyn, P. A., Feiner, C., Cox, C. P., Lipshutz, C. \& COHEN, R. L. (1987) Knowledge about AIDS and highrisk behaviour among intravenous drug users in New York City. AIDS, 1, 247-254.

. Strang, J. S. \& CreEd, F. H. (1985) Treatment of drug dependence, the role of the satellite clinic. Health Trends, 17, 17-18.

Tirrelli, U., Vaccher, E., Carbone, A., Diodata, S., Soria, R., Basio, R., Tombolini, R., Crotti, M., DE Paoli, P., Flego, A., Santini, G. F. \& Monfardini, S. (1986) AIDS Research. (Mary Ann Liebert Inc.) 2, 325-332.

A full list of references is available from the authors.

\title{
Courses in psychotherapy
}

\author{
C. A. Lund, Consultant Psychotherapist, Claremont House, The Royal Victoria \\ Infirmary, Newcastle upon Tyne (Director of the Newcastle Psychotherapy Course)
}

With the rapid expansion in the number and variety of psychotherapy courses in this country, it is perhaps timely to review some of the issues influencing these courses. One purpose of this paper is intended to draw attention to the fact that not only are there interesting conflicts in establishing such courses but also establishments whose power relationships affect the educational effectiveness of these complex teaching ventures. These issues will be discussed with reference to the trainees, trainers, patients, and host organisations.

\section{The trainees}

For trainees the conflicts begin as soon as they contemplate applying for the course. At some level they

This paper is based on a lecture given at the AUTP Conference, 'Teaching Dynamic Psychotherapy' College Oxford 14-16 April 1988. realise that they may be committing themselves to a great deal of work with less time for family and leisure. There is also the risk for career general psychiatrists that they may be 'branded' psychotherapists and that in some circles that may prejudice their progress in psychiatry. More personally, an increase in self-knowledge, whether via a 'training' therapy or other aspects of a course, may be at least uncomfortable.

Most applicants for such courses already, at some level, regard themselves as psychotherapists and stand to suffer a considerable narcissistic blow should they be not accepted on a course. This is felt keenly by them and is compounded by any change, real or imagined, in the eyes of colleagues who may also have looked to them as having psychotherapeutic expertise. This leads one to ask what are the motives for seeking a place on such courses. Is it to learn a technique or range of techniques? Is it a disguised 
way of seeking personal therapy or to be seen to be doing the right thing in a particular culture? Is it to obtain yet more diplomas or to confirm pre-existing self-perceived competence? Or is to train for sustained close interpersonal work of a potentially disturbing nature by means of intensive personal experience? If I seem to be labouring the point it is because, as a course director, one of the hardest tasks I have is informing would-be candidates that they have not been accepted. They all find it hard, become angry and may or may not show that anger openly. Sometimes one is aware that such refusals become a focus of criticism of the course specifically and psychotherapy generally.

Once having become trainees on such a course they soon discover that their misgivings were well founded in terms of time: "It takes over your life for two years" and the effort, especially when combined with normal work and domestic commitments. This is compounded by therapy with longer term patients than previously experienced, the scrutiny of supervision and a personal training therapy crowded into a two year course. This latter pressure can be relieved to some extent by beginning training therapy before the course. Moreover there are conflicting feelings of being one of a number of trainees. On the one hand there is support, on the other, competition. In respect of the latter issue, we in Newcastle have debated at length about whether or not to have a group experience as part of the course. Each trainee is in at least once per week personal training therapy so would group therapy interfere with this? Who would lead it in a small community of psychotherapists where relationships are complicated already because trainees meet not only in context of course, but are often friends and colleagues of some standing? These arguments may not apply to the Tavistock two year course where trainees are strangers and the group may encourage cohesion. There is also an assumption that a group would of itself resolve tensions and facilitate the life of the seminar group. Those of us who have had a group training are less than sure that such facilitation would occur and given that the same group would be meeting for therapy and seminars then other different problems may arise.

\section{The course}

As will be obvious, the nature, content and staffing of a course will be markedly determined by the politics and personalities of a particular situation, issues that will be addressed a little later. But first, what of the conflicts around the notion of a course.

(a) Is the course to be a basic course for which there is much need with many applicants potentially getting something for relatively little input per capita? Is it to be at an intermediate level, where again there is much need but whose intensiveness diverts resources from other areas? Or is it to be a full training?

(b) Is it to be broadly based, teaching a bit of every school, with the risk of the course being about psychotherapy not a training in psychotherapy? Generally, university courses tend to be about subjects, with vocational training being a traditional weakness, which partly explains why higher training in medicine is in the hands of the Royal Colleges. Is the course to teach variants of one model, e.g. individual and group psychoanalytical psychotherapy? But what can be combined in the span of, say, two years? Alternatively, it may teach a single model giving a sound knowledge and technique of individual psychoanalytical psychotherapy or behaviour therapy.

(c) What is the expected level of outcome of graduates of the course? No matter what is said or written, there is often a marked difference of perspective of those involved. Organisers of a course may regard it as basic or introductory, only to discover that both trainees and employing managers regard the qualification in quite a different light. This influences issues of 'pass' and 'fail'. There is a great variation in trainees both at the beginning and end of courses, with the best third overlapping with graduates of more intensive courses, while the worst third are comparable with trainees of other courses at a more basic level.

Then there is a question of the course in relation to other psychotherapy activities in the locality. Particularly in provincial cities, the likelihood is that the course is 'the course', that is, the only available course of any substance. It has to be remembered that in many parts of the country there is no choice for many trainees; others may be able to choose to go elsewhere, but funding for travel is likely to dry up once managers know there is a local course

'The course' therefore not only profoundly influences the style of therapy for a Region but also poses many conflicts in terms of the politics of rival therapists, e.g. inclusion or exclusion of local therapists, or the withdrawal of time and energy from many commitments in order to mount the course. These considerations can be of real concern to those psychotherapists, particularly consultant psychotherapists, who are in some sense charged with a responsibility to encourage the broadest development of a psychotherapeutic approach to psychiatry and related disciplines. The setting up of a course will develop the commitment and skills of some colleagues but sometimes at the cost of a sense of de-skilling and marginalising others. This conflict, whether acknowledged or not, can have a marked influence on the selection of trainers of a course and therefore on the nature of the course itself. In fairness to the trainees it has to be questioned how much wheeling and dealing is tolerable if it interferes with 
the proper functioning of a course in terms of its boundaries, tasks and standards.

An issue often arises at the end of a course which is by no means expected either by trainers or trainees at the beginning of a course. Such an issue is, "How long is a two year course"? This issue can be understood in relation to analytic training courses which, although about four years in duration, in fact only approximate to that and candidates are deemed ready to qualify when supervisors so consider them. In this situation there is considerable flexibility to allow the candidate to develop in their own way and time. This is closely linked to personal development through analysis which is an integral part of such training.

The position is very different in university based courses for at least two reasons:

(a) Universities are used to operating time limited courses and their calendar and regulations are geared to this way of doing things. Doctorate study is more flexible in terms of time but psychotherapy courses are not designed in those terms.

(b) Beginning with Aberdeen, university courses have rarely built personal training therapy into their courses for a variety of reasons:

(i) They were often initially set up as introductory courses for interested psychiatrists who, in the 1960 s and early 1970 s, would have otherwise received no psychotherapy training as part of their general professional development. As such, personal therapy was not considered necessary.

(ii) University Faculties of Medicine or Science might cavil at the idea of personal psychotherapy being part of university regulations.

Under these constraints there is an implicit assumption that all the candidates will be ready to present at the end of two years. However, the reality is that during the 16 years of continuous involvement I have had with three such courses I have yet to experience a course where there is no deferment of at least one trainee. The reasons for such a situation are complex but include the range of intellectual ability in candidates, the variable starting off points of experience in therapy, specific problems in writing essays and in meeting deadlines for handing in case reports, or supervisors not being satisfied with clinical progress. These problems may be purely academic problems but more usually they include or derive from personal feelings, resistances or acting out in relation to conflicts about being scrutinised, judged, leaving the security of the course, becoming a special problem to the course committee, anger about the course or any one of a number of other conflicts.

Given that and the nature of the topic which is the subject of the course, one is immediately up against the issue of personal therapy. As I have indicated, in contrast to analytic courses, two year courses have come slowly toward personal therapy with some destructive and therefore instructive consequences. In Newcastle, from the outset, personal training therapy once per week was built into the course. The training therapists are part of the course committee but do not give feedback to the course committee. Most of the committee are in agreement with this, particularly given the small psychotherapy community in the North East, but this arrangement gives rise to at least two conflicts.

(a) Should therapy be limited to once per week, when two or three per week may be indicated on grounds of clinical need, to facilitate the therapist's clinical and course work or to enable the two year course to be an element in a longer training which, by means of flexible arrangements with London centres, may become a more substantial training over four years, with continuing two or three times per week therapy?

Such a situation often gives rise to overt or covert envy in other trainees who often assume that the person in twice weekly therapy is a favoured candidate, even when there may be indications that more therapy may be needed because of difficulty. This may be displaced onto the course committee who may be attacked for not providing a group experience.

(b) Should therapy stop at the end of two years? The financial arrangements in relation to the course stop after two years but there is an option for private arrangements after that, if so desired. However, it is clear that therapists differ in how they signal the availability of that option. Those therapists whose experience has been in two year courses plus two year therapy seem to have trainees who stop at two years. Those with full analytic training tend to go on longer. This reflects the training of trainers.

\section{The trainers}

Earlier the circular relationship between the nature of a course and its trainers was mentioned. To return to the sensitive topic of trainers: those of a certain generation may remember the enigmatic quotation facing the first chapter of Samson Wright's Textbook of Applied Physiology. It was a quotation of Rabbi Akiba to his favourite pupil, Simeon ben Yochai, "My son, more than the calf wishes to suck does the cow yearn to suckle". Why should anyone add to busy clinical, teaching, administrative, research and family commitments the burden of commitment to a psychotherapy course? Unless, that is, it engages motives deriving from reproduction, generation and nurturance. Unless it engages also issues of identity.

It has often been noted that supervisors are not trained as supervisors. They are trained as therapists 
and become supervisors. There is a process of passing through generations. On the basis of having established one's identity as an individual capable of reliably generating yet another generation, one is enabled by like-minded colleagues to reproduce and nurture professional 'children'.

In some species of therapist the generation cycle is rapid. It is perfectly feasible for a trainee of a two year course to be supervising on the next two year course. That is to say, a trainee from a two year course may be supervising his succeeding generation before a contemporary on a four year course has taken on his/her second training case. Now such a rapid change in status usually only takes place in situations where the number of trained therapists is small relative to commitments. In more favoured situations the reverse applies. The number of hoops of identity checking a would-be supervisor has to go through are prolonged and rigorous. Small wonder that those who can only achieve self-replicating status in 15 years and those who achieve such status in two years occupy different worlds. Yet the need for generational development, the need to nurture, the need to maintain identity are equally strong.

For those whose training is two years, a position as supervisor on a two year course is natural. A two year course, however, also represents an opportunity for four year trained therapists to reproduce much earlier. The handling of such a mating does, however, require some skill. Depending on the relative balance of the trainers involved, both numerical and personal, the possibilities are:

(a) A patchwork quilt course in which each trainer contributes and replicates true to type, especially if the course is a hybrid three years. On the plus side, the trainees get a broad experience but have themselves to integrate the varied, confusing experience, both theoretical and experiential.

In some situations it is possible that the trainers concerned have enough mutual respect to keep their conflicts at bay and for the course to get by. Unhappily, in other settings, the tension is too great, leading to marked differences of opinion and polarisation within the course committee, which in turn affects the trainees.

(b) A two year course which is a cut down version of a four year course. Provided the development of two year supervisors is reasonably similar to four year trainers, it is possible to run a more integrated course in which teaching and supervision is programmed in ways similar to four year analytic training. This leads to a more consistent experience, allowing more thorough development of the trainees, but at the expense of a narrower range of therapy training and the risk that four years seminar work is crammed into two. In order to achieve this situation the course director has to be ruthless in his selection of supervisors and seminar leaders.

\section{Patients}

The relationship of courses to the needs of patients is very variable. Some courses are geared to alleviating the suffering of patients in an immediate and demonstrable way. Other courses, like their counterparts in research, may be about learning a technique, pursuing the truth wherever it may lead in such a way that application to patient care may be somewhat indirect. Most courses oscillate somewhere between these positions. In considering the position of patients in relation to psychotherapy courses we need to consider both the patients who are treated and are part of the subject matter of a course and patients who are treated subsequently by trainees of that course.

Regarding patients on the course, in the first instance this involves the problems and conflicts associated with finding suitable training cases. Should these be brought for discussion with the supervisor by the trainee who is the primary assessor and who presumably has some motivation to help that particular patient? However, there may be problems of inexperience and identification with the patients. Or should the patient be selected by the supervisor, or indeed another supervisor, and passed on to the trainee with a heavy expectation that the trainee take on the case? There is a conflict here between the understandable wish of a trainee to treat over a period of one to three years someone he/she wants to treat versus the need of the Consultant Supervisor or the Clinic to provide a service and that part of that service is to be assessed once and treated and not to be recurrently assessed. One compromise is to provide trainees with three or four case notes from which to choose.

The conflicts do not stop when the patient has been assessed. If the course is an eclectic course, a trainee may begin therapy in one mode and be tempted to change mode part way through therapy. Is this appropriate and what happens to the supervisory alliance in this situation? In those courses which involve long-term frequent therapy, it may become apparent that the patient could appropriately stop therapy some time before the necessary number of sessions has been amassed, leading to some desperation in the trainee and a clinico-ethical conflict.

What of future patients? To date we have little information as to whether the quantum of relief of suffering is greatest from the products of two or four year courses. How does one assess the relative contribution of the impact of a psychodynamically informed general psychiatrist versus an analyst who sees fewer patients but elucidates a problem of human interaction more clearly? 


\section{Relationship to outsiders}

For the purpose of this discussion, 'outsiders' include the external examiner, visiting speakers and the host organisations.

\section{External examiner}

Who is to be the external examiner? Is it to be a widely respected assessor, a widely respected adviser or a 'political' appointment, that is to say a friend of the course house-style?

Having appointed an assessor there is some pressure to so involve him that he is part of the course and, therefore, not external. Yet there is a need to familiarise him with the course, to use him as an adviser to the course, to meet often enough so that he can comment on students' progress both to the committee and to the trainees so that adjustments can be made early enough to help the trainees. It also reduces the feelings of 'end of course execution' at the hands of an unknown judge. There may be also pressure to make him/her the examiner because of conflicted feelings in the course committee about assessing colleagues, supervisees, future colleagues, friends and trainee 'patients'.

\section{Visiting speakers}

Visiting speakers, more than the external assessor, bring to the surface such conflicts as to how much scrutiny can a course stand in fact or fantasy? Is the course committee to be open or protective of its way of doing things? Are the speakers to be invited to be of diverse opinions or of much the same approach, providing shades of opinion within core beliefs congruent with the course?

\section{Host organisations}

Host organisations comprise universities, NHS training establishments or institutes. These organisations raise complex issues which directly affect the degree of autonomy of any course committee.

The case for university based course includes instant respectability, a tangible paper qualification and recognition by funding bodies. It also provides physical facilities and some staff, although their expertise may be of marginal relevance to a course and in time may have a fragmenting effect. The case against a university base is substantial. They can be very expensive, with up to a $90 \%$ 'rake off' for overheads, the control of the course is ultimately in the hands of the head of department, who may or may not have much interest in the aims of the training and who is anyway subject to considerable interdepartmental financial buffeting, so that the course can assume the role of a pawn in a wider game. In such a situation, members of the course committee are not the final arbiters of their affairs and may be always looking over their shoulders. They tend, therefore, to be less direct in their dealing with trainees. This may be compared with a therapist who is not authentically 'with' the patient but the puppet of his supervisor.

NHS based trainings also have respectability, although this tends to be more that of a technical college or polytechnic than a university. As such, the meaning and value of any diplomas may, rightly or wrongly, be suspect. The financial arrangements for such courses are often more satisfactory but is still vulnerable to overall NHS funding. Moreover, there may be pressures on the course committee to accept staff for training who they might otherwise have rejected. In the independent institutes, the course committee has direct control and therefore can relate more directly to trainees. In some ways it can adapt more easily but may be compromised in the degree of adaptation by a perceived need to represent a particular tradition in psychotherapy. In theory it can stop a course more easily but this may threaten identities and livelihoods more than the closure of university NHS courses. The standing of such courses varies, they rarely issue diplomas and, not being part of the official fabric of British life, are ever vulnerable, though showing remarkable resilience.

\section{Conclusion}

The object of this paper has been to bring into the open many of the issues and compromises that attend psychotherapy courses. If the tone of this has been critical it is because only by critical self evaluation can the pioneering efforts of many be carried forward into the next phase of psychotherapy training. It may prove possible to rate local training in such a way that linkages between centres may enable therapists to enhance their skills in an organised way throughout their careers.

\section{Acknowledgements}

I wish to acknowledge colleagues in Aberdeen, Leeds, Newcastle upon Tyne and London upon whose shared experience and comments this paper is based. 